\title{
Criminologie
}

\section{Les prisons de l'an 1984}

\section{André Normandeau}

Volume 9, numéro 1-2, 1976

L’emprisonnement au Québec

URI : https://id.erudit.org/iderudit/017060ar

DOI : https://doi.org/10.7202/017060ar

Aller au sommaire du numéro

Éditeur(s)

Les Presses de l'Université de Montréal

ISSN

0316-0041 (imprimé)

1492-1367 (numérique)

Découvrir la revue

Citer ce compte rendu

Normandeau, A. (1976). Compte rendu de [Les prisons de l'an 1984].

Criminologie, 9(1-2), 232-238. https://doi.org/10.7202/017060ar

Ce document est protégé par la loi sur le droit d'auteur. L'utilisation des services d'Érudit (y compris la reproduction) est assujettie à sa politique d'utilisation que vous pouvez consulter en ligne.

https://apropos.erudit.org/fr/usagers/politique-dutilisation/
Cet article est diffusé et préservé par Érudit.

Érudit est un consortium interuniversitaire sans but lucratif composé de l’Université de Montréal, l'Université Laval et l'Université du Québec à Montréal. Il a pour mission la promotion et la valorisation de la recherche. https://www.erudit.org/fr/ 
LES PRISONS DE

L'AN 1984

André Normandeau

\section{INTRODUCTION}

Un roman célèbre des années 40 présentait l'homme bureaucratisé de l'an "1984" (Orwell) sous un jour excessivement négatif. Heureusement, pour nous, cet homme n'existe pas encore, du moins en 1976, même si certains éléments du portrait d'Orwell sont partiellement présents. La "prévision» est toujours un peu risquée!

En est-il ainsi également des portraits des prisons ou des "non-prisons" de l'avenir que nous présentent depuis quelques années de nombreux auteurs qui ont écrit sur le sujet?

Comme la littérature «pénitentiaire» a subi le phénomène de l'inflation depuis le début des années 70 et qu'au moins une centaine d'ouvrages sur le sujet ont été publiés en langue anglaise ou américaine, nous en avons sélectionné deux qui ont l'avantage de présenter quelques idées contemporaines importantes sur le phénomène de la prison. Le premier, celui de Morris', présente une vision assez fidèle de la perspective des «réformistes» nordaméricains alors que celui de Mathiesen s'inscrit dans la tradition plus radicale des pays scandinaves. Morris veut la disparition d'une partie importante du système pénitentiaire tout en sauvegardant certaines institutions pour les criminels les plus violents, alors que Mathieson ${ }^{2}$ propose la disparition graduelle des prisons d'ici une ou deux générations.

1. Norval Morris (1974) : The future of imprisonment, Chicago. The University of Chicago Press, 144 pages.

2. Thomas Mathiesen (1974): The politics of abolition, Oslo, Norvège. Scandinavian University Books/ ou Londres, Martin Robertson Co., 22 pages. 
Dans cette perspective, l'Office des droits des détenus de Montréal, se rapproche davantage dans son action des points de vue de Mathiesen tandis que le projet de commuanuté thérapeutique du Centre de développement correctionnel (le "C.D.C.m) au sein de l'ancienne Unité spéciale de correction sur le «Campus pénitentiaire» de Saint-Vincent de Paul (Montréal) ressemble étrangement au programme proposé par Morris.

\section{L'OUVRAGE DE MORRIS}

Comme le signale Morris, les prisons ont peu d'amis; personne ou à peu près n'en est satisfait. Des scènes de brutalité, de violence et de conflits raciaux y sont monnaie courante. Et si les prisons doivent "guérir" les criminels, les résultats, avouons-le, sont décevants. Toutefois, l'existence des prisons repose sur d'autres objectifs qui en assurent la survie - que ce soit la punition, l'effet intimidant, la mise à l'écart de personnes jugées dangereuses...

La prison, selon Morris, va donc continuer de survivre. Toutefois, un changement profond de nature «évolutive» est à prévoir.

En pratique, la prison est en soi un pouvoir réel de contrôle utilisé par un État démocratique par rapport à certains de ses citoyens. Pourtant, il n'y a pas de «jurisprudence de l'emprisonnement». Le livre de Morris est une tentative pour définir le rôle spécifique de la prison dans une société démocratique. L'auteur propose de retenir certains principes à la base de ul'idéal de réhabilitation" au sein du système pénitentiaire, tout en dénonçant les effets corrupteurs de programmes de réhabilitation uobligatoires». Morris propose de rendre optionnel ou facultatif le «traitement" en institution.

Des moyens souvent suggérés afin de réduire l'emprisonnement, comme la déjuriciarisation (ou «diversion") et le développement de mesures correctionnelles de nature communautaire, ne seront utiles que si des principes clairs et précis permettent de définir les personnes qui devront finalement «faire» de la prison. Ces principes sont les suivants :

1) Parcimonie : la sanction la moins restrictive (punitive) nécessaire afin d'atteindre les objectifs sociaux connus doit être imposée.

2) Dangerosité : la prédiction d'une criminalité «à venir» doit être rejetée comme base afin de déterminer si oui ou non le criminel doit être emprisonné. 
3) Au mérite: aucune sanction plus grande que celle qui est «méritée» par l'acte criminel le plus récent (ou une série récente d'actes criminels) pour lequel le criminel est condamné.

Les conditions antérieures à l'emprisonnement sont les suivantes :

A. Condamnation par un juge ou un jury ou un plaidoyer de culpabilité pour un acte criminel qui est officiellement puni par l'emprisonnement.

et

B. L'emprisonnement est la sanction la moins restrictive appropriée dans ce cas parce que :

1. toute punition moindre déprécierait la gravité du crime commis;

ou 2. l'emprisonnement de certains criminels qui ont commis des actes semblables à ce cas est nécessaire afin d'atteindre des objectifs intimidants socialement justifiés, et la punition de ce criminel est le moyen approprié pour cette fin;

ou 3. d'autres sanctions moins restrictives ont déjà été appliquées à ce criminel fréquemment dans le passé ou récemment;

C. L'emprisonnement n'est pas une peine qui serait jugée comme "non méritée» ou "excessive» selon les normes et les valeurs de la société ambiante en relation avec le dernier crime ou série de crimes commis.

Dans un dernier chapitre, l'auteur précise finalement le modèle opérationnel d'une institution à sécurité maximale destinée à loger environ 200 criminels violents et récidivistes où le traitement "optionnel" existe sur la base d'un contrat personnalisé entre les autorités et le détenu, où le détenu est averti de la date de sa libération conditionnelle dès son entrée et où des moyens "gradués" de semi-liberté et de liberté deviennent les tests réels de prédiction qui serviront comme guide pour la libération finale.

Si la prison modèle de Morris nous apparaît dans son ensemble comme une proposition valable dont nous nous sommes d'ailleurs inspirés pour le projet du Centre de développement correctionnel à St-Vindent de Paul (Montréal) - nous devons ajouter, cependant, 
que la jurisprudence de l'emprisonnement proposée par Morris ne nous apparaît pas encore suffisamment précise pour déclencher l'adhésion des législateurs et des administrateurs de la justice, ni la mienne d'ailleurs.

\section{L'OUVRAGE DE MATHIESEN}

Mathiesen ne défend pas à tout prix l'idée qu'il faille éliminer les prisons même si son point de conclusion en arrive, à toutes fins pratiques, à préconiser une "politique abolitionniste" (selon son expression) au royaume de l'emprisonnement. Cette conclusion jaillit de deux types de réflexion de la part de l'auteur : une réflexion intellectuelle sur le développement d'une politique du «jamais terminén (unfinished) et une réflexion active à partir d'expériences vécues au sein des associations scandinaves de défense des droits et des conditions de vie des détenus.

Mathiesen opte résolument pour un changement radical et fondamental des structures sociales de sa société. Toutefois, il a acquis la conviction que toute tentative de remplacer l'ordre social existant par un ordre nouveau complètement «terminé mène à la faillite. Aussi désire-t-il, par des recherches actives en particulier, élaborer des grilles de changements sociaux à accomplir tout en remettant "cent fois sur le métier" ces grilles d'action afin qu'elles soient constamment remises en question, un peu comme des "canevas inachevés" qui pousseraient à un perpétuel renouvellement des efforts de l'homme afin de réaliser une justice sociale toujours de plus en plus "égalitaire» pour tous les citoyens sans exception.

L'auteur se lance alors dans la description des activités politiques de groupes de pression scandinaves qui, depuis 1966, se battent énergiquement et systématiquement en faveur des droits des détenus et d'une abolition progressive des prisons pour adultes autant que les institutions pour mineurs. Il s'agit de KRUM (Suède-Association nationale pour la réforme pénale), KRIM (Danemark et Finlande) et KROM (Norvège).

Mathiesen fait une démonstration très serrée des transformations idéologiques profondes qui sont intervenues au sein de ces mouvements au cours de leur développement et de leur structuration. 
En effet, l'idéologie dominante des membres pendant les premières années (1966-1969) était surtout axée sur une politique de réhabilitation et de traitement en milieu institutionnel. Il s'agissait d'améliorer purement et simplement, pour des raisons humanitaires et professionnelles, les conditions de vie des détenus. Peu à peu, devant les échecs retentissants du système pénitentiaire mesurés par les recherches évaluatives menées un peu partout dans le monde occidental, et devant les refus répétés des autorités pénitentiaires de "collaborer" avec eux, ces citoyens engagés ont été amenés à radicaliser leurs actions et à préconiser une politique criminelle orientée vers l'abolition des institutions.

L'auteur démontre, à la lumière des actions de ces associations scandinaves, que si on joue le jeu d'améliorer le système pénitentiaire, on se fait prendre par l'uestablishment» et on ne réussit qu'à mieux faire fonctionner la "machine» sans la changer en profondeur. Aussi propose-t-il un changement radical du système socio-économique qui, selon lui, amènerait automatiquement l'abolition des prisons. En attendant ce changement global il favorise, sur le terrain concret qu'il décrit, soit le monde des prisons, des moyens de "briser ce sous-système social», comme le développement du syndicalisme chez les détenus et l'utilisation spontanée aussi bien que planifiée des grèves de la faim par les détenus, moyens, d'ailleurs, que les associations scandinaves ont effectivement favorisés et développés in praxis depuis quelques années.

Mathiesen revient finalement à son point de départ pour nous prévenir qu'une politique d'abolition des institutions n'est pas suffisante même si elle réussit un jour puisque seule une politique du "jamais terminé» est valable. Si jamais la "révolution de l'univers des prisons" a lieu, il faudra reprendre le combat pour d'autres types d'abolition car l'homme inventera malheureusement toujours d'autres "systèmes répressifs", même en milieu libre, qu'il faudra combattre sans cesse pour la dignité et la liberté de l'homme.

La boucle dialectique de Mathiesen est sans issue. Elle force le criminologue engagé à remettre constamment en question son action.

\section{CONCLUSION}

Il est juste et il est utile que la justice pénale n'ait pas pour seules préoccupations le châtiment des coupables et l'intimidation 
de leurs imitateurs éventuels. En effet, bien souvent, la criminalité apparaît avant tout comme un phénomène d'inadaptation sociale liée à certaines conditions de vie, familiale ou sociale, dont l'intéressé ne peut être tenu pour responsable. En outre, comme la prison n'opère qu'une neutralisation momentanée des criminels, la meilleure protection de la société consiste à tenter de les rendre à leur sortie de prison moins dangereux pour leurs concitoyens qu'ils ne l'étaient à leur entrée.

La justice doit donc, dans une large mesure, selon Monsieur "tout-le-monde» s'attacher à l'amendement, à la resocialisation, à la réinsertion sociale, ou comme on dit plus généralement au "traitement" des criminels. Encore faut-il prendre conscience des limites de cette politique et ne pas accepter de se payer de mots.

Contrairement, en effet, à ce qu'on avait pu en attendre, le fameux traitement du criminel, dont la légitimité est déjà discutable dans la mesure où il implique une investigation de la personnalité du sujet "traité», s'est révélé décevant et il faut rappeler ici les raisons qui font douter aujourd'hui de son efficacité.

En premier lieu, lorsque le milieu social, comme c'est actuellement le cas, est très criminogène, les plus édifiantes leçons de morale administrées dans le milieu artificiel de la prison ont peu de chances de porter longtemps leurs fruits, surtout si les détenus ne reçoivent pas une formation professionnelle leur permettant, après leur libération, de trouver une situation sociale améliorée.

En outre, l'objectif de resocialisation paraît difficilement compatible avec la réalité de l'incarcération, qui rompt les liens que le détenu pouvait avoir avec son entourage, avec sa famille et avec son milieu professionnel. Dès lors, il faudrait, pour amender les criminels, éviter l'emprisonnement dans la mesure du possible - sauf peut-être à étendre l'application du régime de la semi-liberté et à instituer d'autres modes d'exécution des peines privatives de liberté.

Il conviendrait aussi de ne pas verser dans l'illusion qui consisterait à penser que la personnalité humaine demeure indéfiniment malléable. Passé un certain âge, elle s'ossifie comme le squelette. L'effort maximal doit donc être d'éducation plutôt que de rééducation, et il doit être entrepris le plus tôt possible dans le cadre naturel de la famille, dans celui de l'école, dans les foyers de groupe... Mais par la suite, s'agissant d'adultes de 25 ans et 
plus, il est douteux de pouvoir rééduquer durablement et profondément des réflexes souvent acquis dès le plus jeune âge. Le coût social d'une entreprise effective de réducation des criminels menée sur une grande échelle serait donc très élevé, compte tenu du nombre et de la qualification du personnel nécessaire. C'est pourquoi, jusqu'à présent, en matière de resocialisation, les déclarations de principe ont souvent pris le pas sur les réalisations concrètes.

Enfin, le traitement est tout à fait contre-indiqué pour deux sortes de malfaiteurs : ceux qui sont parfaitement bien intégrés dans la société et "normaux" à tous égards, comme la quasi-totalité des délinquants en "col blanc», et ceux qui, comme les gens du «milieu» et les auteurs d'agressions de toutes sortes, constituent une menace inacceptable pour les autres citoyens, et doivent faire l'objet de mesures d'intimidation et de neutralisation.

Il serait donc hasardeux de vouloir fonder sur le "traitement", toute une politique criminelle.

Somme toute, certaines des idées de Morris et, particulièrement, de Mathiesen, pourraient être utiles au criminologue qui serait tenté de prendre pour «la» solution une panacée telle que le «traitement en milieu institutionnel». 